\title{
TENSIONES Y RETOS PARA LA GOBERNANZA AMBIENTAL EN LAS ÁREAS NATURALES PROTEGIDAS. EL CASO DE LA RESERVA DE LA BIÓSFERA SELVA EL OCOTE EN CHIAPAS
}

\author{
Carla Beatriz Zamora Lomelí*
}

\section{RESUMEN}

El modelo de conservación de la biodiversidad en México, enfrenta constantemente el desafío de trabajar con la población que habita en las zonas de amortiguamiento de las áreas naturales protegidas (ANP). Tal es el caso de la Reserva de la Biósfera Selva el Ocote en el estado de Chiapas, donde a través de una investigación cualitativa, se identifican las tensiones y retos para la administración del ANP, frente a un esquema de gobernanza ambiental, que pudiese abonar a la construcción de balances de poder, entre distintos actores sociales vinculados con el propósito de la conservación, atendiendo a las dimensiones sociales y ambientales de la sustentabilidad.

Palabras clave: áreas naturales protegidas, gobernanza ambiental, poder.

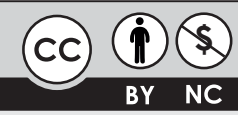

Recibido: 20 de noviembre de 2019 | Aprobado: 22 de febrero de 2020.

\section{CÓMO CITAR ESTE ARTÍCULO:}

Zamora Lomelí, C. B. (2020). Tensiones y retos para la Gobernanza Ambiental en las áreas naturales protegidas. El caso de la reserva de la biósfera selva el Ocote en Chiapas. Collectivus, Revista de Ciencias Sociales, 7(2), 91-106. DOI: https:// doi.org/10.15648/ Collectivus.vol7num2.2020.2675

"Doctora en Ciencias Sociales con Especialidad en Sociología. Investigadora Asociada "C", Grupo de Estudios Socioambientales y Gestión Territorial, El Colegio de la Frontera Sur. Carretera Panamericana y Periférico Sur S/B, Barrio María Auxiliadora, San Cristóbal de Las Casas, Chiapas, CP, 29290, correo electrónico: czamora@ecosur.mx 
$92 \mid$ Tensiones y retos para la gobernanza ambiental en las áreas naturales protegidas.

El caso de la reserva de la biósfera selva el Ocote en Chiapas

\title{
TENSIONS AND CHALLENGES FOR ENVIRONMENTAL GOVERNANCE IN NATURAL PROTECTED AREAS. THE CASE OF THE EL OCOTE JUNGLE BIOSPHERE RESERVE IN CHIAPAS
}

\begin{abstract}
The biodiversity conservation model in Mexico constantly faces the challenge of working with the population that lives in the buffer zones of the natural protected areas (PNA). Such is the case of the selva el Ocote Biosphere Reserve in the state of Chiapas, where through a qualitative research, the tensions and challenges for the administration of the PNA are identified, facing an environmental governance scheme that could contribute to the construction of power balances between different social actors linked to the purpose of conservation, taking into account the social and environmental dimensions of sustainability.
\end{abstract}

Keywords: environmental governance, natural protected areas, power.

\section{Introducción}

En las últimas décadas, se ha evidenciado que el impacto de las actividades humanas, ha ocasionado importantes pérdidas en la biodiversidad debido, entre otras cosas, a la contaminación y degradación de los suelos, la contaminación del agua y el aire, el desvío del agua hacia ecosistemas intensamente gestionados y sistemas urbanos, la fragmentación del hábitat, la explotación selectiva de especies, y el agotamiento del ozono estratosférico (Gittay, Suárez y Watson, 2002), de tal manera que estamos ante una crisis ecológica que presenta procesos de degradación ambiental de alcance global (Solana, 2007,p.202).

En respuesta, la política ambiental derivada de los acuerdos internacionales sobre cambio climático, se institucionalizó en México con esquemas para la adaptación y mitigación del cambio climático, con base en los servicios ecosistémicos, la sustentabilidad y la importancia de áreas destinadas a la conservación ambiental. En este contexto, entre finales de la década de los setenta y mediados de los noventa, se estableció un esquema de conservación de la biodiversidad que paulatinamente, ha ido incorporando la perspectiva social a través del establecimiento de áreas naturales protegidas (ANP), clasificadas en distintas categorías de acuerdo con su función para la conservación en:

Zonas del territorio nacional sobre las que la nación ejerce su soberanía y jurisdicción, en donde los ambientes originales no han sido significativamente alterados por la actividad del ser humano o que requieren ser preservadas y restauradas y están sujetas al régimen previsto en la Ley. (LGEEPA, 2012, p.2)

Sin embargo, el trabajo de conservación implica reconocer una amplia gama de actores que intervienen en el proceso, desde administradores gubernamentales hasta los propios habitantes que convienen en territorios declarados como ANP. En este sentido, el modelo mexicano de conservación considera que es "posible instituir ANP sin desplazar a la población residente en ecosistemas particularmente valiosos (Gil, 2019, p.23) lo cual implica enfrentar retos entre las necesidades de reproducción social y la normatividad que rige las ANP. 
El objetivo de este trabajo, es identificar algunos de los desafíos y tensiones desde la dimensión social para las ANP, a partir del análisis de los actores con respecto al territorio en el contexto de un área natural protegida: la Reserva de la Biósfera Selva el Ocote (REBISO), ubicada en el estado de Chiapas, México. Este trabajo, deriva de la investigación interdisciplinaria llamada "vulnerabilidad social y biológica ante el cambio climático en la Reserva de la Biosfera Selva El Ocote", el cual fue financiado de 2013 a 2016, por el Consejo Nacional de Ciencia y Tecnología y que, para los propósitos de la investigación desde el enfoque de las ciencias sociales, ha permitido el seguimiento del trabajo con posterioridad a la conclusión del proyecto de investigación referido.

El documento se estructura en tres partes; la primera, presenta una revisión del estado del arte en torno a las áreas naturales protegidas, en particular sobre lo escrito en torno al estudio de caso que aquí se presenta. La segunda, describe la metodología cualitativa que fue utilizada para el levantamiento de datos en campo y se enfatizan los niveles de análisis desde las percepciones y las relaciones de poder entre actores, lo cual se presenta de manera detallada en el tercer apartado, mientras que en el apartado de discusión y valoración de hallazgos se reflexiona en torno a la construcción de la gobernanza ambiental con base en los resultados. Finalmente, en la tercera se concluye que los principales retos sociales para la conservación, se encuentran anclados en la estructura social, económica y cultural de las poblaciones ubicadas en las zonas de amortiguamiento de la REBISO y su abordaje requiere un enfoque de interculturalidad ${ }^{1}$ para el equilibrio de la balanza de poder en el territorio.

\section{Estado de la cuestión}

Resulta indudable la importancia de la conservación ambiental y en particular, las áreas naturales protegidas, como estrategia ante la crisis ecológica, dada su función para salvaguardar la biodiversidad y proveer de servicios ecosistémicos a nivel global. En México, la política ambiental ha adoptado tal estrategia a través de la declaración de ciento setenta y seis áreas naturales de carácter federal, bajo la administración de la Comisión Nacional de Áreas Naturales Protegidas (CONANP) en nueve regiones del país (CONAP, 2019).

En torno a ello, se han escrito innumerables trabajos con enfoques desde diferentes disciplinas y esfuerzos interdisciplinarios importantes, aunque de manera sobresaliente, las investigaciones sobre el impacto en términos de conservación de la biodiversidad, son más frecuentes desde las ciencias naturales (Carabias, 2007; Sarukhan, 2008; Villalobos, 2009), mientras que los enfoques desde la dimensión social son más recientes (Betancour et al. 2014; Brener, 2010; Durand y Jiménez, 2010).

De manera particular, en el caso del estado de Chiapas, desde las ciencias sociales se han escrito trabajos que dan cuenta de las tensiones al interior de las ANP, tal es el caso de los trabajos compilados en el libro "Paradojas en tierras protegidas: democracia y política ambiental en reservas de la biósfera en Chiapas" (Legorreta, Márquez y Trench,

\footnotetext{
${ }^{1}$ Entendida en el sentido de Walsh (2009) como "complejas relaciones, negociaciones e intercambios culturales, y busca desarrollar una interacción entre personas, conocimientos, prácticas, lógicas, racionalidades y principios de vida culturalmente diferentes; una interacción que admite y que parte de las asimetrías sociales, económicas, políticas y de poder, y de las condiciones institucionales que limitan la posibilidad de que el "otro" pueda ser considerado sujeto con capacidad de actuar" (Walsh, 2009, p.45).
} 
94| Tensiones y retos para la gobernanza ambiental en las áreas naturales protegidas.

El caso de la reserva de la biósfera selva el Ocote en Chiapas

2014), donde se discuten los desafíos para la democracia ambiental, en contextos de inequidad social y política como ocurre en este estado del sureste de México.

Por su parte, Tejeda (2009), aborda la dimensión de los conflictos sociales para el caso particular de la Selva Lacandona, un espacio con una historia de colonización particular en el estado de Chiapas, que por su potencial biodiverso y sus procesos sociales ha llamado la atención de numerosos investigadores e investigadoras como Cano, 2018; Cruz y Rosano, 2006; Durand, Figueroa y Trench, 2012, entre otros.

En particular, sobre el caso que nos ocupa, la Reserva de la Biósfera Selva el Ocote, la mayoría de los trabajos se concentran en aspectos de biodiversidad y sustentabilidad como Gómez et al. (2012), quienes realizaron un estudio, para estimar la viabilidad de la producción sustentable desde la ganadería en algunas comunidades del ANP; mientras que Orantes et al. (2013), analizaron el aprovechamiento de los recursos naturales en la comunidad de Emilio Rabasa, una de las más cercanas a la zona núcleo de la REBISO.

De manera más reciente, a partir del proyecto del cual deriva este trabajo, en 2017, se editó el libro Vulnerabilidad social y biológica ante el cambio climático en la Reserva de la Biosfera Selva El Ocote (Ruiz, Gordillo, Ramírez y Cruz, 2017), que reúne una compilación de trabajos de corte biológico y de análisis de procesos sociales, en un esfuerzo interdisciplinario para comprender las dimensiones de la vulnerabilidad; sin embargo, no existen investigaciones que analicen las tensiones, conflictos y retos en el marco de las relaciones de poder entre actores en la REBISO, tal como el que aquí se presenta.

\section{Metodología utilizada}

Para el abordaje metodológico, se recurre al estudio de las percepciones ambientales enfocado en quienes habitan la zona de amortiguamiento de la Reserva. El enfoque de percepciones plantea una relación holística entre naturaleza y sociedad donde, lejos del determinismo geográfico, el ambiente ejerce influencia sobre la percepción de las personas, es decir, "las personas y sus procesos individuales y sociales son moldeados por las condiciones ambientales del lugar donde viven. Por esta razón, es fundamental estudiar procesos como las percepciones dentro de los contextos ecológicos donde ocurren las interrelaciones de las personas con su ambiente" (Benez, Kaufer y Álvarez, 2010, p.136).

Se trata de un enfoque basado en la subjetividad de los individuos, sus discursos y actitudes que están mediados por sus atributos genéricos, etarios y étnicos, pero también culturales, sociales y políticos, "de esta manera el concepto de percepción ambiental articula los aspectos individuales y colectivos, los contextos, los sentires y comprensiones, para la consideración de los puntos de vista locales que permitirán la construcción de diálogos" (Sánchezy Martínez, 2015, p.600).

Por otra parte, si bien el concepto de percepción tiene fundamentos en la filosofía y la psicología, paulatinamente se ha convertido en un concepto interdisciplinario que conjuga a la antropología, la sociología y las ciencias ambientales para dar cuenta de procesos sociales complejos.

En congruencia con el enfoque metodológico descrito, el diseño de la investigación recurrió al uso de herramientas cualitativas como las entrevistas a profundidad (22), la observación participante y los grupos focales (4). Asimismo, se cumplimentó una encuesta a ochenta y seis personas ${ }^{2}$ en cuatro comunidades ubicadas en la REBISO: Veinte Casas, Emilio Rabasa, Nuevo San Juan Chamula y San Joaquín El Rosario.

\footnotetext{
${ }^{2}$ Aplicada sobre un universo de 110 personas mayores de edad, en cuatro comunidades de la zona
} 
En conjunto, los datos generados fueron procesados para generar diversas categorías de análisis, sobre percepciones ambientales utilizando software Nvivo ${ }^{\circledR}$, además de realizar el estudio de redes con apoyo de UCINET $6{ }^{\circledR}$.

\section{Descripción y análisis de resultados}

Ubicada en la cordillera de la Sierra Madre de Chiapas, la Reserva de la Biósfera Selva El Ocote, abarca 101 288-15-12.5 hectáreas entre los municipios de Ocozocoautla, Cintalapa, Tecpatán y Jiquipilas en el Estado de Chiapas, en el sureste de México,

$\mathrm{Su}$ importancia, radica en que contiene muestras representativas de selva alta perennifolia y mediana subperennifolia, así como numerosas especies de importancia económica (maderables, medicinales, comestibles y ornamentales). Para el caso de vertebrados, en este refugio se han reportado un total de 646 especies de vertebrados terrestres. Por otro lado, el complejo y variado sistema cavernario existente en la Selva "El Ocote" es un refugio perfecto para algunos animales, pues constituyen ambientes limitados (SEMARNAT, 2001, p.5).

Figura 1. Mapa de ubicación de la REBISO en Chiapas

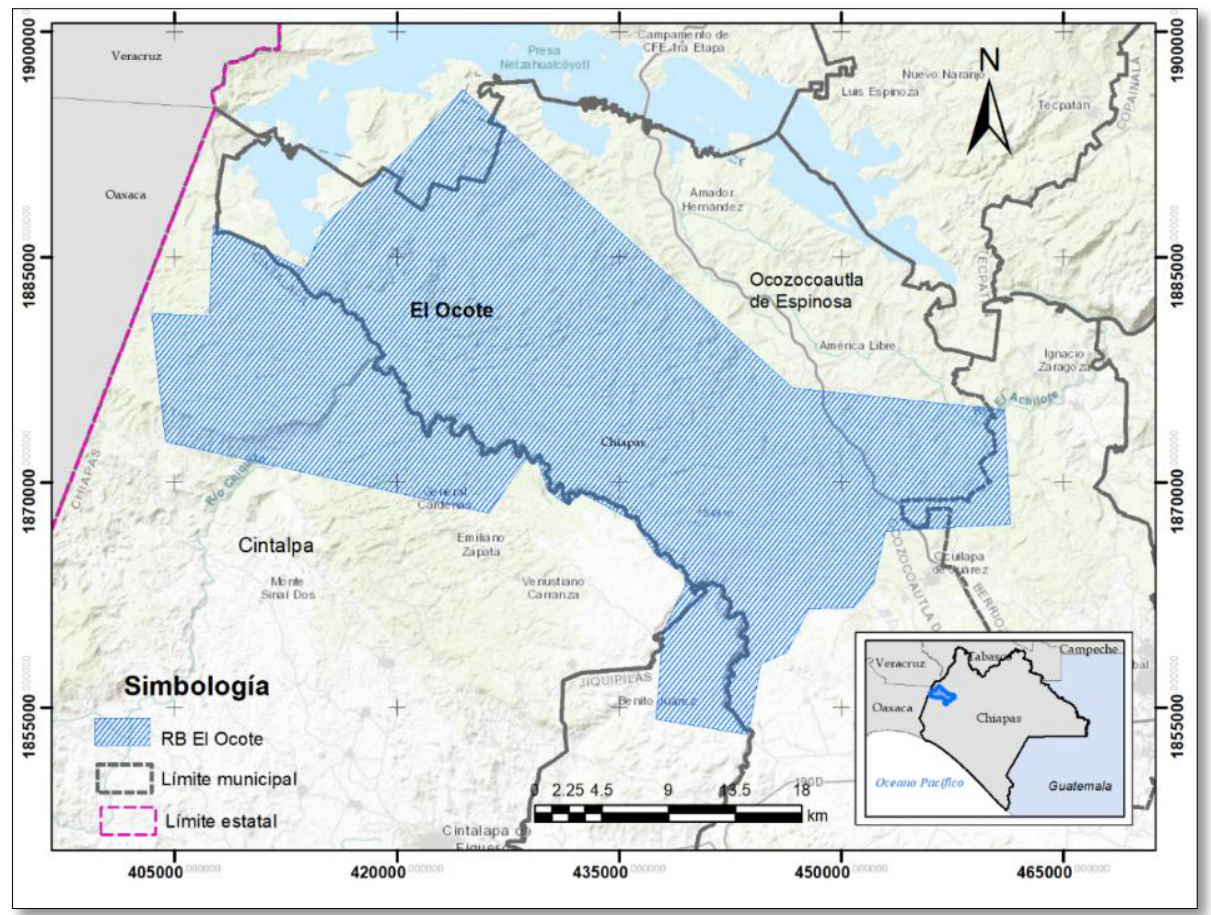

Fuente: Laboratorio de Información Geográfica y Estadística, El Colegio de la Frontera Sur, 2019.

de amortiguamiento, con un margen de error de 5\% e intervalo de confianza de $95 \%$. La encuesta consistió en preguntas cerradas sobre percepciones ambientales. 
96| Tensiones y retos para la gobernanza ambiental en las áreas naturales protegidas.

El caso de la reserva de la biósfera selva el Ocote en Chiapas

Cabe mencionar, que, si bien no existen registros actualizados sobre la población, el dato más reciente estima que aproximadamente ocho mil diecisiete personas (Cano, 2019 , p.244) habitan en treinta y dos comunidades dentro de la zona de amortiguamiento, de las cuales el $72 \%$ de los habitantes son indígenas tsotsiles, seguido de indígenas zoques y habitantes mestizos. Estas localidades tienen una propiedad de la tierra ejidal, con excepción de dos comunidades que se rigen bajo la propiedad comunal de la tierra.

Se trata de comunidades que tienen como actividad principal el trabajo agrícola y pecuario donde la práctica de ganadería extensiva, ha representado una amenaza para los fines de la conservación, toda vez que se realizan desmontes de bosques caducifolios para dar lugar a los pastizales (SEMARNAT, 2001), aunque existen mecanismos para regular tales prácticas e incluso se han desarrollado alternativas desde la ganadería intensiva sustentable (Gómez et al. 2012), persiste "un conflicto ambiental y productivo, ya que tiene poca tierra de potencial agropecuario y no se aprovecha de manera óptima, aunado a la presión de escasez de tierra, se desmontan terrenos no aptos para las actividades agropecuarias" (op. cit.), lo cual también se relaciona con el crecimiento poblacional de alrededor del $4 \%$ anual, que demanda mayor presión sobre la tierra.

Buena parte la población que habita en la REBISO es relativamente joven, es decir, son grupos con no más de tres o cuatro generaciones de haber llegado a la zona, por lo que, si bien existe un proceso de reterritorialización, siguen apropiándose del espacio. La relación entre naturaleza y sociedad no ha sido sencilla, pues al inicio las personas que llegaron en el proceso de colonización, tuvieron que conocer nuevos animales y aprender a trabajar con terrenos pedregosos. Las propias redes, que mantienen las personas han posibilitado la migración hacia Estados Unidos y estados del norte de México, de manera cíclica, entre marzo y abril llegan a las comunidades los camiones que trasladarán a los hombres a otros espacios para trabajar como jornaleros agrícolas.

Por otra parte, los antecedentes de la declaratoria de la Reserva se remontan a 1972, cuando se emitió un primer decreto estatal por 10.000 hectáreas categorizándola como Área Natural y Típica del Estado de Chiapas, tipo ecológico bosque lluvioso alto: Posteriormente, en 1982, se declaró como Zona de Protección Forestal y Fáunica Selva El Ocote con 48.000 hectáreas y finalmente en el año 2000, se decretó a nivel federal como Reserva de la Biósfera con el reconocimiento de la UNESCO (2006), ampliando su extensión a 101.000 hectáreas (SEMARNAT, 2001).

Al margen de las declaratorias del espacio para la conservación, la historia del territorio tiene antecedentes de población previos a los decretos de la política ambiental, lo cual es uno de los motivos por los que hay presencia de conflictos y tensiones de manera cotidiana entre los pobladores de la Reserva y las limitaciones de la política de conservación, como se expondrá más adelante.

Por otra parte, los antecedentes históricos del territorio dan cuenta que hasta el siglo XV, de nuestra era, la etnia Zoque ocupó todo el occidente de Chiapas, territorio que fue disminuyendo paulatinamente durante los siglos siguientes debido a la llegada e invasiones de otros grupos (op. cit.), por lo tanto, ha sido un territorio habitado en distintos periodos históricos por diversos grupos indígenas y mestizos.

En la época más reciente, desde 1968, las poblaciones que ahora forman parte de la zona de amortiguamiento, tienen su origen en la dinámica del reparto agrario en Chiapas, donde pobladores de comunidades de la zona de los Altos demandaron tierra al gobierno federal, la cual fue concedida de manera gradual desde la década de los sesenta en el espacio que hoy comprende la REBISO, y que en algunos casos eran tierras nacionales o 
terrenos de ranchos, que fueron cediendo a través de procesos de compraventa y que, según narraron algunos entrevistados, fueron fundados por personas que habían sido contratadas por rancheros para trabajar en la ganadería en Ocozocuautla o en los trabajos de construcción de la presa Malpaso y poco a poco llegaron en busca de tierra, un recurso que se encuentra sobreexplotado en los Altos, zona donde existe mayor densidad poblacional y donde en promedio los productores, poseen menos de media hectárea de tierra.

En suma, el proceso de colonización se resume de la siguiente manera:

Hacia los años 1950, se menciona la presencia de ranchos ganaderos que se abastecían de mano de obra de poblaciones tsotsiles de Los Altos, así como la atracción de población de este grupo étnico hacia finales de esta década, cuando se inició la construcción de la presa Nezahualcóyotl, localmente conocida como presa Malpaso. Al parecer, parte de la población tsotsil que se empleó como mano de obra para la construcción de esta presa, tras la inundación de las tierras decidió asentarse en la región, aunque en otros casos la llegada de familias tsotsiles estuvo relacionada con las expulsiones de población protestantes desde Los Altos, hacia los años 1970. De modo, que desde entonces se inició un proceso de colonización y de solicitud de ejidos y nuevos centros de población ejidal, hoy en día ubicados en la parte norte y suroeste del polígono de conservación, además de la titulación de pequeñas propiedades ubicadas mayoritariamente al este de la reserva. Dentro de este panorama, para 1996, se reportaba además la ocupación de terrenos nacionales, así como la presencia de terrenos baldíos. En estos primeros años de constituidas las Reservas de la Biósfera, el trabajo de gestión de dichas áreas tardó en tomar ritmo, así como las poblaciones tardaron en saber $y / 0$ en aceptar que se encontraban dentro o en inmediaciones de un polígono de conservación la relación entre el personal a cargo y las poblaciones locales estuvo marcada por mucha tensión, concretamente expresada en amenazas a los funcionarios que se atrevieran a ir a las localidades para hablar de la conservación. (Cano, 2019, pp. 252-253)

Así, buena parte de los primeros asentamientos derivados de la migración intermunicipal han tenido lugar entre 1968 y 1994, es decir, en algunos casos fueron espacios concedidos por dotación ejidal con anterioridad al decreto de Reserva. De esta forma, los pobladores de la zona han tenido que empatar una forma de vida rural, vinculada a los decretos y restricciones que impone la normatividad ambiental, lo cual como se verá más adelante, resulta en distintas áreas de oportunidad para empatar la correspondencia entre la población y su territorio desde las relaciones naturalezasociedad.

Sin embargo, "la baja productividad característica del sector rural se correlaciona con los niveles extremos de pobreza que predominan particularmente en las zonas sur, sureste y oriente del país, estableciéndose un círculo vicioso de pobreza y conductas de supervivencia que impactan de manera negativa sobre el capital ecológico de la nación" (SEMARNAT,2001, p.34).

La pobreza en la que vive el $85 \%$ de las personas que habitan en la zona, es un problema estructural que se ha combatido de manera parcial, por las instancias de gobierno y las organizaciones no gubernamentales que llegan a la REBISO. A pesar de los esfuerzos por canalizar programas, el desarrollo sustentable se convierte en un estandarte mientras los hombres de las comunidades continúan migrando hacia Estados Unidos y otros estados en el norte de México, porque en sus lugares de origen no tienen los suficientes medios económicos para revertir su condición de pobreza. En ello coincide la Comisión Nacional de Derechos Humanos: 
98 Tensiones y retos para la gobernanza ambiental en las áreas naturales protegidas.

El caso de la reserva de la biósfera selva el Ocote en Chiapas

Conforme a los datos publicados por el CONAPO, aproximadamente el $84 \%$ del total de localidades ubicadas dentro de las áreas protegidas tienen índices de marginación alto y muy alto, situación que contrasta con la gran riqueza biológica y el potencial existente de aprovechamiento sustentable con los recursos naturales en las mismas. (CNDH, 2016, p.72).

En palabras del director de la REBISO, Roberto Escalante, las comunidades siguen estando abandonadas, y es curioso porque a estas alturas del tiempo, deberían ya ser comunidades con un poquito más oportunidades. Irónicamente, y así se lo decimos a la gente de las comunidades: ustedes viven dentro de un área con mucha riqueza y mucha pobreza. (R. Escalante, comunicación personal, 12 de mayo de 2015).

Por lo tanto, son comunidades cuyo ejercicio de derechos fundamentales es muy limitado, pues carecen de los derechos básicos a la salud, la alimentación y la vida digna, lo que implica que su capital social ${ }^{3}$ es limitado con respecto a la posibilidad de decidir sobre el territorio que habitan.

Es necesario entender que:

Un ANP es, antes que nada, un proceso de territorialización, que, si bien tiene en su centro una calificación jurídica, importa porque es resultado de la interacción entre un conjunto de actores que, a lo largo del tiempo, producen formas específicas de apropiación material y simbólica que, en su conjunto, configuran (o más bien: reconfiguran) un territorio. (Gil, 2019,p.31)

Por lo tanto, confluyen una gama de actores diversos con nivel de relaciones diferentes para decidir con respecto al territorio, dado que "el territorio es un concepto muy claramente vinculado con las relaciones de poder, está vinculado siempre con el poder y con el control de procesos sociales mediante el control del espacio" (Haesbaert, 2013, p.13), por ello, el manejo del área natural protegida debe garantizar que los mecanismos de administración del espacio consideren los derechos agrarios, ambientales, económicos y sociales de los actores junto con las condiciones institucionales para la gestión ambiental en el marco de las relaciones de poder, entendiendo que "el poder tiene que ser analizado como algo que circula, o más bien, como algo que no funciona sino en cadena" (Foucault, 1980, p. 152), es decir:

El poder es ante todo una relación de gobierno entre dos o más actores, por tanto, el poder aparece como el arte de A para conducir o estructurar el campo de acción de B, manteniendo este último un margen de opciones para oponer una contraofensiva hacia el primer. (Guerra, 1999, p.116)

Lo anterior, ocurre si consideramos el ANP como territorio para la conservación en donde confluyen múltiples actores, donde en principio es un espacio normado jurídicamente, pero implica la correlación con quienes habitan el mismo espacio, quienes ejecutan proyectos de intervención social o quienes realizan labores de investigación académica en el territorio y ello presenta distintos gradientes de relaciones sociales de poder, como se verá más adelante.

\footnotetext{
${ }^{3}$ Entendiendo, el capital social como concepto aglutinador que combina "confianza y normas de reciprocidad, redes y formas de participación civil, y reglas formales e informales o instituciones articulados en el marco de la acción colectiva" (Ostrom, 2003, p.155).
} 
Por ello la importancia de comprender que:

El escenario actual de la conservación en las áreas naturales protegidas se caracteriza porque las instituciones ambientales y las poblaciones locales parten de bases epistémicas distintas, las cuales acompañan las acciones y discursos sobre el medio natural. Desde luego, la conservación requiere generar espacios de entendimiento mutuo que propicien la toma de decisiones pertinentes y negociadas. Una argumentación en pro del diálogo intercultural requiere de condiciones favorables y un nuevo lenguaje con base en ideas compartidas, en las cuales se reflejen las distintas concepciones de la naturaleza y del mundo (...) La participación ciudadana tendría que ser el eje central de la política ambiental y debería enmarcarse en un contexto donde coincidan los más diversos intereses, sin embargo, la política ambiental mexicana refleja más las percepciones, visiones e intereses de los tomadores de decisiones que los de la población local, lo cual repercute en el fracaso o éxito de un programa o de las políticas públicas. (Bello y Estrada, 2012, pp. 220-221).

Como se ha referido, por su naturaleza jurídica y la importancia que representa el territorio para la conservación, en las áreas naturales protegidas confluyen una amplia gama de actores.

Para los propósitos de este trabajo se identificaron a los siguientes actores:

1) Institucionales. Responsables de administrar y ejecutar la legislación ambiental en el espacio y quienes a su vez ejecutan la política que emana de los acuerdos internacionales con respecto al cambio climático.

2) Infra institucionales. Donde se identifican a los organismos internacionales que observan e inciden en la definición de las políticas públicas ambientales a nivel federal y estatal, éstos pueden además financiar los proyectos locales que pretenden implementar el desarrollo sustentable.

3) Académicos. Profesionistas especializados con intereses de investigación particularmente biológica, y a su vez integrantes del Consejo Técnico Asesor de la Reserva.

4) Organizaciones no gubernamentales (ONG). Existen algunas organizaciones cuyo trabajo se ha concentrado en temas de sustentabilidad y conservación que trabajan en algunas comunidades de la Reserva, como el caso de Altépetl, A.C., Regal Springs México, Conservación Biológica y Desarrollo Social A.C. y FONCET A.C. entre muchas otras.

5) Fundaciones. Refiere a las organizaciones que financian proyectos de conservación, para este caso, se tiene registro.

La Agencia Internacional de desarrollo (AID), The Nature Conservancy (TNC), Comunidad Económica Europea (CEE), World Wildlife Found (WWF), Klamath National Forest (KNF-USFS), SEMARNAT, National Fish and Wildlife Foundation (NFWF), Fish and Wildlife Service (FWS), ARBORDAY Foundation, CONMPTON INC., Industrias BIMBO, Fondo Mexicano para la Conservación de la Naturaleza (FMCN) y el Gobierno del estado de Chiapas (SEMARNAT, 2001, p. 5).

6) Comunitarios, ejidales y avecindados. Refiere a la población que habita en el polígono de la reserva y cuyos procesos históricos de colonización del territorio, les confieren características culturales y sociales particulares donde la identidad indígena tsotsil y zoque prevalece, aunque todos tengan sus orígenes en algún pueblo indígena, pero a través de generaciones se haya perdido la identidad y cultura. 
100| Tensiones y retos para la gobernanza ambiental en las áreas naturales protegidas.

El caso de la reserva de la biósfera selva el Ocote en Chiapas

Del análisis de redes entre los actores descritos, se obtiene lo siguiente:

\section{Figura 2. Redes y relaciones entre actores}

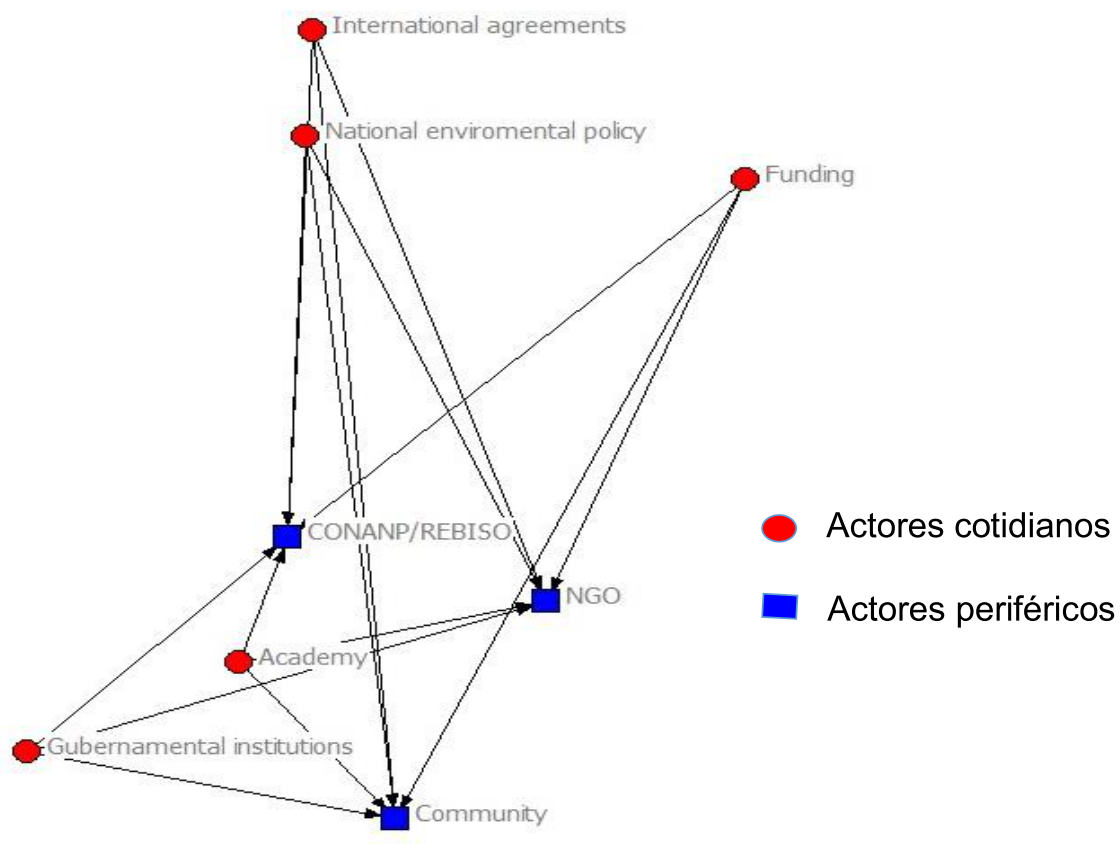

Fuente: presente investigación.

La gráfica anterior, se encuentran a nivel superior dos actores infrainstitucionales, es decir, que son un conjunto de agentes que definen la política ambiental a nivel internacional, estableciendo acuerdos generales de donde derivan acciones para la adaptación y mitigación del cambio climático. Su ubicación en ese espacio, refleja la distancia con respecto a la operación y sobre todo, las comunidades, que se encuentran en el punto medio inferior. En el plano superior derecho, se observa a las fundaciones que financian proyectos de conservación, también distantes de las comunidades ${ }^{4}$.

Los actores simbolizados con cuadros azules, en la gráfica (comunidades, CONANP/REBISO y Organizaciones No Gubernamentales) se clasificaron como actores cotidianos, en tanto queremos ilustrar el margen de acción en el campo de la ANP de manera regular, mientras que los actores simbolizados con círculos rojos son aquellos cuya presencia en el territorio, es poco constante o solamente normativa como el caso de los tomadores de decisión en el plano de la política ambiental nacional e internacional.

\footnotetext{
${ }^{4}$ Conviene mencionar, que cuando se habla acerca de las comunidades, se hace como una categoría descriptiva genérica, no analítica, dado que cada localidad rural tiene una dinámica comunitaria propia que de forma analítica impediría su homogeneización.
} 
En el plano de relaciones cercanas se encuentran los actores de la academia, ONG y CONANP/REBISO, que actúan de manera coordinada en una alianza interesada en apoyar el trabajo de los administradores de la Reserva, tanto en términos de la conservación como en el trabajo con la población que habita las zonas de amortiguamiento.

Estas alianzas, se concretan en el Consejo Asesor Técnico, pero también apoyando a la institución con la formulación de proyectos de investigación que dotan de información científica para la toma de decisiones; sin embargo, la relación entre este conjunto de actores con las comunidades es instrumental, es decir, se tiene poca incidencia sobre la mejora en las condiciones de vida de las personas que ahí habitan, por las propias limitaciones del trabajo que realizan y la complejidad de la realidad de quienes viven en la zona, que como se mostró líneas atrás, se encuentra en condiciones de pobreza y marginación, que le llevan a buscar estrategias de sobrevivencia dentro y fuera de la zona.

En suma, los retos más claros se encuentran en la distancia entre las comunidades que están en la base de esta red de actores y los actores institucionales como los financiadores de proyectos, los académicos y con las definiciones de la política a nivel de régimen nacional e internacional.

En complemento, del estudio sobre las percepciones de los pobladores, las personas encuestadas consideran, en su mayoría, que encuentran más dificultades que beneficios al vivir en las condiciones de un área natural protegida, como se observa en la siguiente gráfica:

Figura 3. Percepciones de pobladores con respecto al ANP

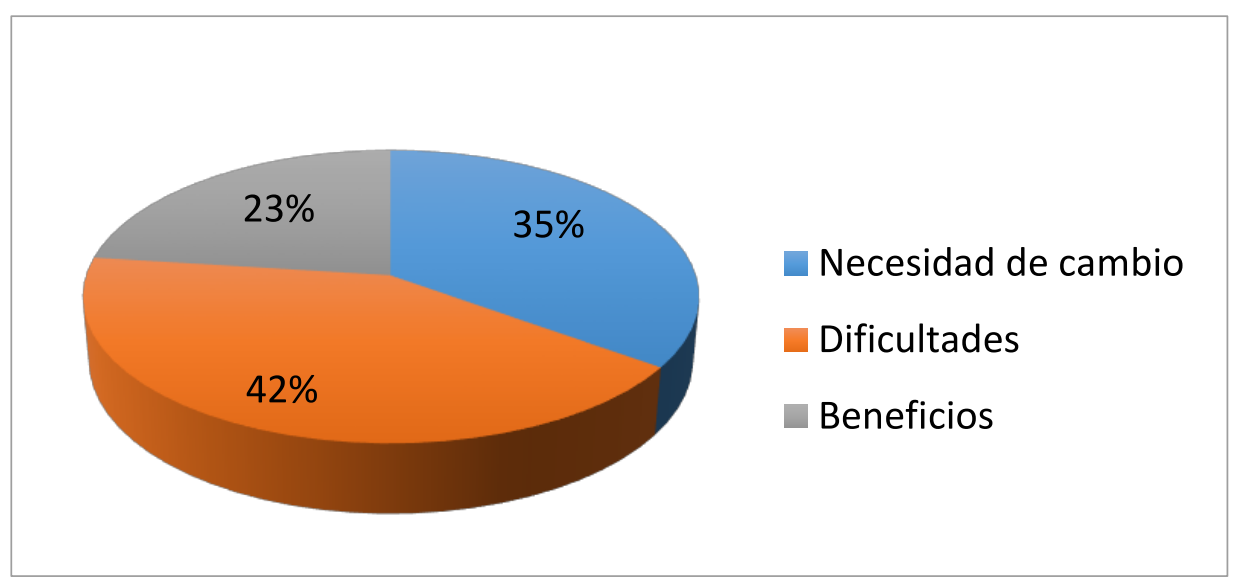

Fuente: presente investigación.

A partir de los datos, se encuentra que la principal tensión se relaciona con la cuestión económica vinculada a la precariedad en las comunidades, en este sentido, las personas que viven en la REBISO manifestaron que la principal preocupación es la falta de acceso a recursos económicos, según refirió el $87 \%$ de las personas encuestadas; mientras que del mismo universo, el $75 \%$ consideró también que las dificultades propias del cambio climático como la ampliación del perio do de sequía o la pérdida de cosechas por fenómenos hidrometeorológicos, son cada vez más constantes, a pesar de vivir en una ANP, lo cual representa una tensión para garantizar la producción agrícola. 
102 Tensiones y retos para la gobernanza ambiental en las áreas naturales protegidas.

El caso de la reserva de la biósfera selva el Ocote en Chiapas

Otra de las tensiones que las personas perciben de manera cotidiana, se relaciona con la normatividad que rige al área natural protegida (65\%), en este sentido, varias personas expresaron sentir temor de ser encarcelados si transgredían alguna de las regulaciones, como por ejemplo, cortar un árbol para reemplazar las tablas con que están construidas las paredes de su casa, pero también en las entrevistas perciben que hay una distancia entre quienes diseñan las políticas ambientales y la realidad en las comunidades, como ilustra el siguiente testimonio:

"Las dependencias, traen lineamientos que se hacen desde la oficina, que no es lo mismo estar en la oficina que estar aquí, no nos dejan opción, o lo toman o lo dejan, hay necesidades de la familia que obligan a tomar programas que se ofrecen, aunque esos no tienen sentido y no sirven”. (Grupo focal, comunicación personal, San Joaquín, Ocozocuautla, 10 de noviembre de 2015)

Finalmente, el $67 \%$ de las personas encuestadas refirió que la convivencia cotidiana con animales silvestres también es un problema cuando algunas especies invaden las cosechas, lo cual se vincula con la tensión respecto a la vigilancia de la normatividad ambiental; mientras que la preocupación por el crecimiento demográfico y la dotación de tierra es otra tensión que las personas perciben con preocupación, dado que la legislación establece límites a la frontera agrícola y no es posible expandirla más, mientras las familias siguen creciendo y requieren de más tierra.

Por otra parte, los beneficios de vivir en la zona de amortiguamiento del ANP percibidos por las personas, se relacionan principalmente con la apreciación del paisaje y la calidad de vida en la convivencia con un ambiente limpio (92\%), además de una visión de sustentabilidad a futuro para que el territorio conserve la riqueza de su biodiversidad $(80 \%)$.

Asimismo, las comunidades muestran interés en trabajar con proyectos de desarrollo sustentable, lo que podría ser un reto para la gobernanza ambiental, lo cual representa una oportunidad para la operación de los proyectos de sustentabilidad, que se destinan a las comunidades, aunque también se perciben limitaciones, como ilustra el siguiente testimonio:

"Desafortunadamente, no se recibe ningún apoyo para conservar, en mi parcela a lo mucho hay una hectárea y le comentaba a mis hijos que si no nos daban recursos íbamos a tirar 3 hectáreas de montaña, si nosotros no tenemos un beneficio vamos a tirar la montaña porque no tenemos donde trabajar, con hambre y nada en el estómago no se puede conservar, hay pocos programas para conservar, nadie nos motiva a seguir conservando y nos gustaría tener viviendas dignas y vivir bien". (Grupo focal, comunicación personal, San Joaquín, Ocozocuautla, San Joaquín, 10 de noviembre de 2015)

En otros términos, los retos en la operación de los proyectos ambientales se enfrentan con una forma de ejercer el gobierno a través de mecanismos asistenciales y clientelares, por lo que muchas veces las personas beneficiarias están acostumbradas a recibir proyectos y beneficios sin compromisos de largo plazo, lo que puede representar una amenaza a los proyectos de conservación si no se impulsa el desarrollo de las capacidades de quienes habitan en la reserva, sobre todo, se distancia del ideal de la gobernanza ambiental al omitir el desequilibrio de poderes en la toma de decisiones ambientales. 


\section{Discusión y valoración de hallazgos}

Un concepto que abona para la discusión de los resultados es el de gobernanza ambiental, concebida como el "proceso de formulación y refutación de imágenes, diseños y ejecución de los procedimientos y prácticas que configuran el acceso, control y uso de los recursos naturales entre actores diferentes" (Castro, Hogenboom y Baud, 2015, p.18) y que, a través de distintos mecanismos, pueden influir en las decisiones ambientales. Aunque dicho enfoque, carece de la dimensión del poder y del conflicto entre actores, por lo que aquí se propone incorporar ambas dimensiones de análisis a fin de entender las relaciones entre actores y la balanza de poder en torno a las decisiones de política ambiental en los territorios de las ANP.

En este sentido, las diferencias de poder, raciales y de clase (Elias y Scotson, 2015), inciden las posibilidades de consolidar los procesos de gobernanza, toda vez que quienes cuentan con mayor capital social (profesionistas urbanos, trabajadores académicos o funcionarios estatales) se concentran en atender la dimensión de la conservación de biodiversidad, objetivo de las ANP, más que en la búsqueda de alternativas económicas y sociales para el mejoramiento de la vida de las personas que habitan en el mismo territorio.

Otro aspecto importante, es la gobernanza ambiental como un eje de políticas públicas, la diferencia cultural, étnica y lingüística de las poblaciones que habitan en los territorios de las ANP y la relación con los operadores de las instituciones como la CONANP.

Ello derivado de lo observado en el trabajo de campo, por ejemplo, en el marco de la recomendación general número 26 de la $\mathrm{CNDH}$, tuvo lugar en 2017, la consulta a los pueblos indígenas con respecto al manejo de la REBISO; sin embargo, dicha consulta cumplió parcialmente con los criterios internacionales para informar en su propia lengua a los habitantes del territorio sobre los mecanismos de manejo para la conservación, al preguntar a los operadores sobre la importancia de considerar a esta población de acuerdo con los fines del ANP, respondieron: "para qué preguntarles si ni siquiera son de aquí [refiriendo a la colonización de la zona]" (R. Díaz, comunicación personal, 12 de julio de 2018), lo que indica una negativa al reconocimiento de la diversidad cultural en el ANP, y podría ser un punto de conflicto entre los actores institucionales y la población.

Es importante referir también, que en la REBISO se identifica el territorio con mayor conflictividad social relacionado con actividades de la delincuencia organizada, en palabras de un operador:

"El conflicto de Chimapalas ha deforestado mucho, hace quince años el sitio estaba arbolado, pero la falta de presencia institucional que controle la deforestación ha hecho que se realice la deforestación. También la zona del Encajonado siempre ha sido tierra de nadie, hay organizaciones delictivas de narcotráfico conectado con el cártel del Golfo y con robo de ganado, no es fácil entrar ahí". (Grupo focal REBISO, comunicación personal, 15 de marzo de 2019)

En este sentido, la gobernanza ambiental enfrenta serios límites para la participación de la base comunitaria que no podrán atenderse hasta ampliar la mirada con herramientas de las ciencias sociales, que permitan observar la dimensión del conflicto social y las relaciones de poder en las ANP. Conviene así, considerar distintos modelos de conservación que (Gil, 2009) distingue en tres tipos:

La primera, se podría denominar comunitarismo antiestatista, es la más resistente a la creación de las ANP desde los órganos del estado, ya que sostiene que son las comunidades que habitan en las ANP, frecuentemente asociadas con identidades indígenas, quienes deberían tomar en sus manos la tarea de la sustentabilidad, sin más intervención del 
104 Tensiones y retos para la gobernanza ambiental en las áreas naturales protegidas.

El caso de la reserva de la biósfera selva el Ocote en Chiapas

estado que la garantía de los derechos de propiedad sobre la tierra y otros recursos naturales. En el otro extremo, está la postura que llamamos estatismo duro, que propone una intervención estatal sobre las áreas de alta concentración de biodiversidad, que las convierta en propiedades públicas y busque alguna manera de compensar a las poblaciones locales para que encuentren otro lugar donde vivir. La tercera postura, que llamamos estatismo flexible, pugna por un liderazgo estatal en la constitución de las ANP, acompañado de un conjunto de iniciativas que conviertan a las comunidades residentes en aliados de la conservación (p.39-40).

Así, los hallazgos de este trabajo se inclinan hacia la constitución de un modelo de estatismo flexible vinculado a la gobernanza ambiental, siempre que se atiendan lo que aquí se ha identificado como tensiones en el plano económico, y normativo, e incorporen una perspectiva de género (Esteban, et al., 2019), atendiendo a un esquema de educación ambiental en diversos niveles educativos (Iñigo, 2019).

\section{Reflexiones finales}

En este trabajo, se considera que el estatismo flexible acompaña los procesos de gobernanza ambiental y puede ser una vía importante siempre que permita un equilibrio en la balanza de poder con respecto al territorio, incorporando a las personas que habitan en las comunidades de la ANP desde la diversidad cultural y la búsqueda de alternativas conjuntas a los problemas estructurales en términos económicos y sociales.

Ello requiere una estrategia institucional, vigilante de los derechos a la información y los derechos económicos, sociales y culturales de las personas residentes en las ANP. El rescate del conocimiento local, ofrece una ruta para incrementar el capital social de lo que aquí se ha nombrado comunidades para aludir a quienes habitan en las zonas de amortiguamiento de la REBISO.

Resulta también importante, atender a la operación de la Reserva con una óptica regional, si bien se identifica a las zonas desde su vocación para la conservación, una lectura desde las ciencias sociales incrementaría la posibilidad de incidencia considerando procesos históricos, sociales y culturales propios de cada comunidad.

Por último, y no menos importante, la construcción de la gobernanza ambiental requiere esfuerzos importantes a nivel institucional y estructural para disminuir la brecha de las desigualdades sociales y la pobreza en la que vive la población que habita en las ANP, para lo cual se necesita más que un proyecto de desarrollo sustentable o una beca gubernamental. Es necesario, que los pobladores sean sujetos actuantes de su propio desarrollo y de su relación con el medio ambiente en el que viven.

\section{Referencias bibliográficas}

Bello, E., y Estrada, E. (2012). Cultura, conservación y áreas naturales: hacia una conservación incluyente. En Silva, G. y Parra, M. Patrimonio cultural y natural, desde los enfoques de la sustentabilidad y del saber local. (219-240) México: Asociación Mexicana de Estudios Rurales, A.C., Universidad Nacional Autónoma de México.

Benez, M., Kauffer E., y Álvarez, G. (2010). Percepciones ambientales de la calidad del agua superficial en la microcuenca del río Fogótico, Chiapas. Frontera Norte, 22 (43) 129158.

Cano, I. (2018). De montaña a "reserva forestal": Colonización, sentido de comunidad y producción de la conservación ecológica en el sureste de la Selva Lacandona, México. México: Instituto de Investigaciones Sociales, UNAM. 
Cano, I. (2019). Las ANP Federales de Chiapas. En Comisión Nacional de Derechos Humanos \& Universidad Nacional Autónoma de México (2019). Estudios sobre el cumplimiento e impacto de las recomendaciones generales, Informes especiales y pronunciamientos de la CNDH (2001-2017) Tomo VI. Areas naturales protegidas y Derechos Humanos. Coordinación de Humanidades, UNAM-CNDH. Recuperado de http://www.cndh.org.mx/sites/all/doc//Informes/Especiales/ANP-DH.pdf

Carabias, J., Arriaga, V., y Cervantes-Gutiérrez, V. (2007). Las políticas públicas de la restauración ambiental en México: limitantes, avances, rezagos y retos. Botanical Sciences, (80S), 85-10.

CNDH. Comisión Nacional de Derechos Humanos. (2019). Recomendación general número 26 sobre la falta y/o actualización de programas de manejo en Áreas Naturales Protegidas de carácter federal y su relación con el goce y disfrute de diversos derechos humanos. Recuperado de Comisión Nacional de Áreas Naturales Protegidas, Las áreas naturales protegidas en México, Recuperado de https://www.gob.mx/conanp

CONANP. Comisión Nacional de Áreas Naturales Protegidas. (2019), Áreas naturales protegidas de México. Recuperado de https://www.gob.mx/conanp\#1692.

Cruz, C. T., y Rosano, C. M. (2006). Apropiación territorial y aprovechamiento de recursos forestales en la comunidad Frontera Corozal, Selva Lacandona, Chiapas, México. Revista de Geografía Agrícola, (37), 79-95.

DOF. Diario Oficial de la Federación. (2012). Ley General del Equilibrio Ecológico y Protección al Ambiente (LGEEPA). Reforma actual. México. Recuperado de http://biblioteca.semarnat.gob.mx/janium/Documentos/Ciga/agenda/DOFsr/148.pdf

Durand, L., y Jiménez, J. (2010). Sobre áreas naturales protegidas y la construcción de nolugares: Notas para México. Revista líder, 16(12) 59-72.

Durand, L., Figueroa, F., y Trench, T. (2012). Inclusión, exclusión y estrategias de participación en áreas protegidas de la Selva Lacandona, Chiapas. La naturaleza en contexto. Hacia una ecología política mexicana, 237-267.

Elias, N., y Scotson, J. (2015). Establecidos y marginados: Una investigación sociológica sobre problemas comunitarios. México: Fondo de Cultura Económica.

Esteban, M., Amador, L.V., Mateos, F., y Olmedo, F.J. (2019). Mujer y medio ambiente. Una aproximación desde la acción socioeducativa Collectivus, Revista de Ciencias Sociales, 6(1), 177-195. DOI: https://doi.org/10.15648/Coll.1.2019.10

Figueroa, F., y Calzada, L. (2018). Gobernanza del manejo de recursos forestales en México en: Sistemas socioecológicos. Marcos analíticos y estudios de caso en Oaxaca, Veronique Avila y M. Perevochtchikova, coords. 205- 225. México: Instituto de investigaciones económicas, UNAM.

Foucault, M. (1980), Microfisica del poder. Madrid: Ediciones La Piqueta.

Gil, M. (2019). Introducción general. El contexto histórico. En Comisión Nacional de Derechos Humanos y Universidad Nacional Autónoma de México. Estudios sobre el cumplimiento e impacto de las recomendaciones generales, Informes especiales y pronunciamientos de la CNDH (2001-2017) Tomo VI. Áreas naturales protegidas y Derechos Humanos. Coordinación de Humanidades, UNAM- CNDH. Recuperado de http://www.cndh.org.mx/sites/all/doc//Informes/Especiales/ANP-DH.pdf.

Gittay, H., Suárez, A., y Watson, R. (coords.). (2002). Cambio Climático y Biodiversidad. Documento Técnico V Del Ipcc. Grupo Intergubernamental de Expertos sobre el Cambio Climático. Recuperado de https://archive.ipcc.ch/pdf/technical-papers/climate-changesbiodiversity-sp.pdf. 
106 Tensiones y retos para la gobernanza ambiental en las áreas naturales protegidas.

El caso de la reserva de la biósfera selva el Ocote en Chiapas

Gómez, H., Guevara, F., Hernandez, M., Nahed, J., Rodríguez, L., y Pinto, R. (2012). Analysis of Cattle Raising and Institutional Perspectives Regarding Collective Action in the El Ocote Biosphere Reserve, Chiapas, Mexico. Journal of Animal and Veterinary Advances 11 (6) 831-840.

Haesbaert, R. (2013). Del mito de la desterritorialización a la multiterritorialidad, Revista Cultura y representaciones sociales, 8 (15) 9-42.

Iñigo, L. (2019). Educación para la preservación del medio ambiente. Collectivus, Revista de Ciencias Sociales, 6(1), 215-234. DOI: https://doi.org/10.15648/Coll.1.2019.12

Guerra, E. (1999). El problema del poder en la obra de Michel Foucault y Norbert Elias. Estudios sociológicos, 17 (49) 95-120.

Legorreta, M., Márquez, C., y Trench, T. (2014) Paradojas de las tierras protegidas. Democracia y politica ambiental en reservas de biosfera en Chiapas. México: Universidad Nacional Autónoma de México, Centro de Investigaciones Interdisciplinarias en Ciencias y Humanidades, Centro Regional de Investigaciones Multidisciplinarias, Universidad Autónoma Chapingo.

Orantes, C., M. Pérez, C. del Carpio y C. Tejeda (2013). Aprovechamiento del recurso maderable tropical nativo en la comunidad de Emilio Rabasa, Reserva de la Biosfera Selva El Ocote, Chiapas, México. Madera y Bosques. (19) 7-21.

Ostrom, E., \& Ahn, T. K. (2003). Una perspectiva del capital social desde las ciencias sociales: capital social y acción colectiva. Revista mexicana de sociología, 65(1), 155233.

Ruiz, L., Gordillo, G., Ramírez, N., y Cruz B. (Eds.). (2017). Vulnerabilidad social y biológica ante el cambio climático en la Reserva de la Biosfera Selva El Ocote. México: El Colegio de la Frontera Sur.

Sánchez, S., y Martínez, Y. (2017). Percepciones de agricultores tsotsiles sobre el clima, variabilidad climática y sus cambios en la localidad "Veinte Casas", Reserva de la Biosfera Selva El Ocote, Chiapas. En Ruiz, L., Gordillo, Á., Ramírez, N., y Cruz, B. (Eds.). Vulnerabilidad social y biológica ante el cambio climático en la Reserva de la Biosfera Selva El Ocote. (pp. 593-622) México; El Colegio de la Frontera Sur.

Sarukhan, J. (Ed.). (2008). Capital natural de México. México. Comisión Nacional para el Conocimiento y Uso de la Biodiversidad (CONABIO).

Secretaría de Medio Ambiente y Recursos Naturales, Comisión Nacional de áreas Naturales Protegidas. (2001). Programa de Manejo de la Reserva de la Biosfera Selva El Ocote. Recuperado de http://www.conanp.gob.mx/Que_Hacemos/Pdf/Programas_ Manejo/Ocote.Pdf.

Solana, L. (2007). Antropología social y medio ambiente: sobre la necesaria articulación entre ecología cultural, ecología de sistemas, ecología política y etnoecología. En Garrido, F. González de Molina, J. y Solana, L. (eds.), El paradigma ecológico en ciencias sociales, (pp. 201-226) Barcelona: Icaria Antrazyt.

Villalobos, I. (2009), Áreas naturales protegidas: instrumento estratégico para la conservación de la biodiversidad. Gaceta Ecológica, (54). 24-34.

Walsh, C. (2009). Interculturalidad crítica y educación intercultural. Construyendo interculturalidad crítica, 75-96. 